\title{
后疫情时代全球可持续发展目标加速行动的推动
}

\author{
冯思远 ${ }^{1,2}$,赵文武 ${ }^{1,2}$, , 华 廷 ${ }^{1,2}$,王 涵 ${ }^{1,2}$ \\ 1 北京师范大学地理科学学部地表过程与资源生态国家重点实验室, 北京 100875 \\ 2 北京师范大学地理科学学部陆地表层系统科学与可持续发展研究院, 北京 100875
}

摘要: “SDGs 加速行动” 是国际组织、政府部门、私营机构和其他利益敒关方为加快落实 2030 年可持续发展议程采取的全球行 动。2019 年联合国可持续发展目标峰会后, 政府、国际组织、私营部门等提出了 214 项 SDGs 加速行动。2019 年爆发的新型冠 状病毒肺炎 (Corona Virus Disease 2019,COVID-19) 对实现可持续发展目标带来了系列影响,后疫情时代如何推动全球 SDGs 加 速行动的实施成为重要的问题。对可持续发展评估报告(2019)和可持续发展目标加速行动等政策文件进行信息提取,建立加 速行动匹配性指数模型和各国应对新冠疫情的恢复力指数模型, 根据匹配性-恢复力分类体系将各国按照 17 项可持续发展目 标分为 9 类,为推动后疫情时代全球可持续发展目标加速行动提供支撑。研究发现: (1) 现有可持续发展目标加速行动的实施 与区域需求不匹配, 且这种不匹配的情况在 COVID- 19 爆发前已经出现; (2) 加速行动的实施受限于现有可持续发展水平和国 家经济基础, 区域关注的可持续发展目标与其自然地理位置和社会发展水平有着密切的关系,多边组织机构和其他利益做关方 需要在发展中国家大力推动可持续发展加速行动; (3) 下一步实施加速行动需要加强国际间的合作, 根据分类框架和可持续发 展目标的关联关系, 分重点推进加速行动的实施, 完善可持续发展指标监测体系, 分类设立后疫情时代不同时期的阶段目标, 分 阶段循序渐进,定期反馈追踪, 以在 2030 年促进 17 项可持续目标的实现。

关键词: 可持续发展目标加速行动; 可持续发展目标;可持续发展;COVID-19;

\section{How to implement SDGs Acceleration Actions in the post-epidemic era}

FENG Siyuan ${ }^{1,2}$, ZHAO Wenwu ${ }^{1,2, *}$, HUA Ting ${ }^{1,2}$, WANG Han ${ }^{1,2}$

1 State Key Laboratory of Earth Surface Processes and Resource Ecology, Faculty of Geographical Science, Beijing Normal University, Beijing 100875, China 2 Institute of Land Surface System and Sustainable Development, Faculty of Geographical Science, Beijing Normal University, Beijing 100875 , China

\begin{abstract}
SDGs Accelerated Actions are a series of global actions taken by international organizations, government departments, private institutions, and other stakeholders to accelerate the implementation of the 2030 Agenda for Sustainable Development. After the United Nations Sustainable Development Goals Summit in 2019, a total of 214 SDGs Accelerated Actions were put forward by various stakeholders. The Corona Virus Disease 2019 ( COVID- 19) outbreak in 2019 has a series of impacts on the achievement of the SDGs. How to implement SDGs Acceleration Actions in the postepidemic era has become an important issue. This paper extracts information from the Sustainable Development Report 2019 and the SDGs Accelerated Action policy, and establishes a matching index model and resilience index model to explore the matching of SDGs Accelerated Action and the ability of countries to recover from COVID- 19. According to the matchingresilience classification system, countries are divided into 9 categories to provide support for implementing SDGs Acceleration Actions in the post-epidemic era. The study found that: (1) the currently implemented SDGs Acceleration Actions did not match the regional needs, and the mismatch occurred before the COVID- 19. (2) The implementation of
\end{abstract}

基金项目: 国家自然科学基金项目 (42042026) ; “一带一路”国际科学组织联盟资助( ANSO-SBA-2020-01)

收稿日期:2020-10-08; 采用日期:2021-08-01

* 通讯作者 Corresponding author.E-mail: zhaoww@ bnu.edu.cn 
SDGs Acceleration Actions was limited by the current level of sustainable development and economic foundation, and the sustainable development goals of regional concern were closely related to its natural geographical location and development level. Multilateral organizations and other stakeholders need to vigorously promote SDGs Acceleration Actions in the developing countries. (3) The focus of the SDGs Acceleration Actions in the next step was to strengthen international cooperation, promote the implementation of accelerated actions based on the classification framework and the nexus relationship of SDGs, improve the sustainable development indicator monitoring system, establish stage goals of different types countries for different periods in the post-epidemic era, and regular feedback and track to promote the realization of SDG1- 17 in 2030.

Key Words: SDGs Acceleration Actions; Sustainable Development Goals ; sustainable development ; COVID-19

2015 年,联合国举行了可持续发展峰会, 通过了《改变我们的世界: 2030 年可持续发展议程》 (Transforming our world: the 2030 Agenda for Sustainable Development), 正式宣布了 17 项可持续发展目标 (SDGs,Sustainable Development Goals) ${ }^{[1]}$, 该议程旨在用 15 年的时间,在全球实现 17 项可持续发展目标 ${ }^{[2]}$ 。 这 17 项可持续发展目标是对 2000 年联合国千年发展目标 (MDGs, Millennium Development Goals) 的继承 ${ }^{[3]}$ 。 然而,经过四年的发展, 由于致命冲突、气候变化、经济增长不平衡等原因, 人类在可持续发展领域所取得的已 有成果面临着倒退的威胁 ${ }^{[4]}$ 。为了及时改变当前莬境,不同国家政府、国际组织等领导人,在 2019 年联合国 可持续发展目标峰会上一致通过了题为“为可持续发展行动与成就的十年做好准备” (Gearing up for a Decade of Action and Delivery for Sustainable Development) 的政治宣言,承诺进一步提振雄心和动力,筹集资金、加强实 施力度, 按时、按计划实现可持续发展目标 ${ }^{[5]}$ 。由各国政府、组织机构和其他利益敒关方提出的为加快落实 2030 年可持续发展议程自愿采取的具体措施或者承诺, 被称为 “可持续发展目标加速行动” (SDGs Acceleration Actions) ${ }^{[6]}$ 。截至 COVID-19 疫情爆发前, 各国政府、组织机构和其他利益做关方共提出了 141 项 可持续发展目标加速行动,但可持续发展目标加速行动是否与可持续发展水平相适应, 加速行动与区域需求 的关联性均不明确。

2019 年,全球爆发了新型冠状病毒肺炎(COVID- 19,Corona Virus Disease 2019),截至 2021 年 7 月 28 日, 全球确诊病例 1.95 亿,死亡 418 万,生产活动大规模减少,国家经济受到重创,使人类在推动实现可持续发展 目标的道路上进一步偏离了既定的轨道 ${ }^{[7]}$ 。有关实现 17 项可持续发展目标的联合国进展报告指出, 受 COVID- 19 的影响,数以千万计的人民很可能重新陷人极端贫困和饥饿之中 ${ }^{[8]}$ 。COVID- 19 导致的经济损失 加剧了不平等问题, 2020 目标守卫者报告显示, 这次疫情对女性、少数种族和少数民族群体造成了更严重的 影响; 在全球范围内,无偿护理工作整体需求的增加导致女性面临更多负担,同时女性也面临更严重的失业问 题 ${ }^{[9]}$ 。相关研究表明 COVID- 19 对生态环境短期内存在积极的影响 ${ }^{[10-12]}$, 如 COVID-19 大流行期间人类活动 的停止降低了 $\mathrm{CO}_{2}$ 排放 ${ }^{[13]}$ 。但随着经济的复苏, $\mathrm{CO}_{2}$ 排放会恢复原有水平 ${ }^{[14]}$ 。总体而言, COVID- 19 进一步 滞后了 2030 年可持续发展目标的整体实现。

目前关于可持续发展目标的研究多集中在可持续发展目标间的关联 ${ }^{[15-21]}$ 、可持续发展指标体系构 建 ${ }^{[22-23]}$ 、可持续发展目标定量监测 ${ }^{[24]}$ 上,相关研究对可持续发展目标加速行动关注较少, 而可持续发展目标 加速行动是各国对可持续发展目标促进行为的直接表征, 是研究可持续发展目标关联的有效手段; 在研究方 法上,部分学者利用政策文件,探讨了可持续发展目标与政策文件间的关系 ${ }^{[25-27]}$,他们通过梳理政策文件和 可持续发展目标的关联来进一步提出政策的改进建议, 以促进可持续发展目标的实现。然而,这些研究的关 注点主要集中在单个目标的实现,既没有考虑整个目标体系,也没有与区域可持续发展现状相结合。 $\mathrm{Fu}$ 等人 提出需要从系统的角度出发, 理清 SDGs 之间的逻辑关系, 识别不同类型国家的优势和不足, 明晰推动实现 SDGs 在空间和时间上的尺度效应 ${ }^{[28]}$, 且目标间、国家间处于远程耦合的状态 ${ }^{[29]}$, 故基于全球尺度进行政策 文件的分析有利于识别国别间、目标间的异质性。在后疫情时代,由于疫情导致的经济衰退和人口死亡, 需要 
对可持续发展目标的推动有所侧重, 在推行更多加速行动之前,应进一步明晰加速行动与区域需求的关联性。 但目前缺乏对可持续发展目标加速行动与当地可持续发展水平之间匹配性问题的研究,如果不明确可持续发 展目标加速行动实施的数量与当地可持续发展水平的匹配性关系, 就会出现不均衡的分配现象。本文从全球 可持续发展目标加速行动政策文件的角度进行分析,利用可持续发展目标加速行动的数量作为区域对促进相 关可持续发展目标的响应,将联合国 2019 年发布的可持续发展评估报告 ${ }^{[30]}$ 的结果作为各国可持续发展现状 的表征,探讨加速行动的空间分布与区域可持续发展现状的匹配情况,结合各国受新冠疫情的影响程度,将各 国根据分类框架按照 17 项可持续发展目标进行分类,结合可持续发展目标之间的关联关系, 为推动后疫情时 代全球可持续发展目标加速行动提供支撑。

\section{1 数据来源与研究方法}

\section{1 数据来源}

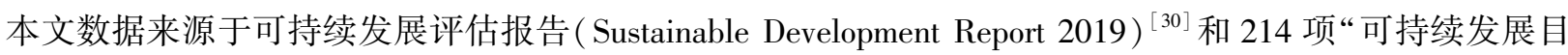
标加速行动” 文件 ${ }^{[6]}$ 。可持续发展评估报告主要提取了 162 个国家可持续发展水平得分和可持续发展目标 得分。“可持续发展目标加速行动” 文件共提取了 214 项“可持续发展目标加速行动” 的受益国和相应加速行 动的受益目标。COVID-19 数据来源于世界卫生组织( WHO, World Health Organization)、霍普金斯大学, 所有 数据采集的时间节点截至 2020 年 8 月 28 日。

1.2 加速行动匹配性指数 (SDR)

截至 2020 年 8 月 28 日,各国政府、组织机构和其他利益做关方共提出了 214 项可持续发展目标加速行 动, 每项加速行动都可以同时使一个或多个可持续发展目标和国家受益, 考虑到这种一对多关系, 本文统计了 214 项 SDGs 加速行动的受益目标和受益国。为便于定量化分析各国加速行动数量与区域需求的关联性, 本 文构建了匹配性指数模型, 以各国 17 项可持续发展目标得分离最优得分 (100 分) 的差距作为区域需求的表 征,外部对该国家特定可持续发展目标对应实施的加速行动数量作为供给的表征, 即 “供给与需求” 比率 (SDR,Supply-Demand Ratio)。SDR 公式如下:

$$
\begin{aligned}
& \operatorname{SDR}_{i j}=\frac{Y_{\text {Num_actions } i j_{i j}}}{Y_{100-\text { Score }_{i j}}} \\
& Y_{\text {Num_actions } i j_{j}}=100 \times \frac{X_{\text {Num_actions } i j_{i j}}}{N} \\
& Y_{100-\text { Score } i j_{i j}}=100 \times \frac{X_{100-\text { Score }_{i j}}}{N}
\end{aligned}
$$

公式 (1) 是匹配性公式, $\mathrm{SDR}_{i j}$ 是第 $i$ 个国家在第 $j$ 个可持续发展目标下加速行动的匹配性指数, $\mathrm{SDR}=1$ 表示匹配性最佳, 供需相等; $\mathrm{SDR}>1$, 表示加速行动数量大于区域需求, 供大于求; $\mathrm{SDR}<1$, 表示加速行动数量 小于区域需求,供小于求。式中, $i$ 是 162 个国家, $j$ 是 17 项可持续发展目标; $Y_{\text {Num_actions }_{\mathrm{ij}} \text { 是Num_actions }}$ 位序标

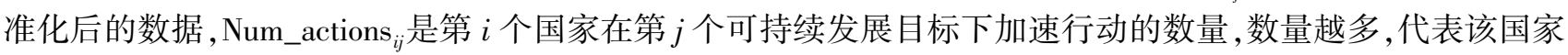

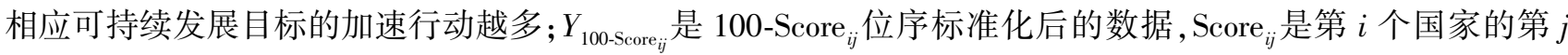
个可持续发展目标得分, Score i $_{i j}$ 满分 100 分, 得分越高, 代表第 $i$ 个国家的第 $j$ 个可持续发展目标发展水平越 好, 100-Score ${ }_{i j}$ 是第 $i$ 个国家的第 $j$ 个可持续发展目标得分与 100 分的差值, 差值越大, 代表对于可持续发展目 标加速行动的需求越大; 公式(2) (3) 是位序标准化的公式形式, 公式(2) 中, $Y_{\text {Num_actions } i j_{i j} \text { 是Num_actions }}$ 位序标

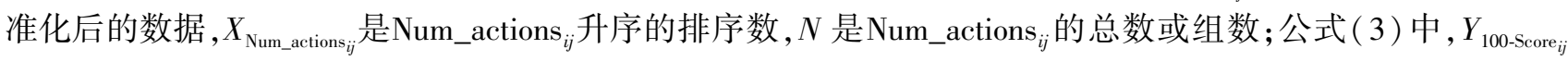

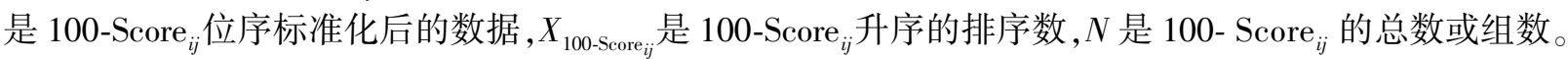

\section{3 恢复力指数 $(R)$}

本文统计了截至 2020 年 8 月 28 日 162 个国家对应的可持续发展水平得分、每十万人的 COVID- 19 感染 
率。为分析各国应对 COVID- 19 的恢复能力, 本文通过构建恢复力指数模型,建立以各国家可持续发展水平 作为抗击 COVID- 19 的前提条件和物质基础,COVID- 19 感染率作为受灾程度的恢复力指数,揭示在原有国家 基础上从 COVID- 19 中恢复的能力, 定量化研究 162 个国家恢复力。恢复力 ( $R$, Resilience) 公式如下:

$$
\begin{aligned}
& R_{i}=\frac{Y_{\text {Score }_{i}}}{Y_{P I_{i}}} \\
& P I_{i}=\frac{\text { Cumulative_cases }_{i}}{\text { Population }_{i}} \\
& Y_{\text {Score }_{i}}=100 \times \frac{X_{\text {Score }_{i}}}{N} \\
& Y_{P I_{i}}=100 \times \frac{X_{P_{i}}}{N}
\end{aligned}
$$

式中, $R_{i}$ 是第 $i$ 个国家的恢复力指数, $R=1$ 表示恢复力匹配; $R>1$, 表示恢复力越好; $R<1$,表示恢复力越差; 式 中, $i$ 是 162 个国家, $Y_{\mathrm{Score}_{i}}$ 是 $\mathrm{Score}_{i}$ 位序标准化后的数据, $\mathrm{Score}_{i}$ 是第 $i$ 个国家可持续发展水平得分,得分越 高,代表该国可持续发展水平越高,抗击抗击 COVID-19 的前提条件和物质基础越好; $Y_{P I_{i}}$ 是 $P I_{i}$ 位序标准化化 后的数据, $P I_{i}$ 是第 $i$ 个国家每十万人的 COVID-19 感染率, 感染率越高, 受灾程度越严重。公式 (5) 是感染率 公式 (PI, Prevalence of Infection), Cumulative_cases ${ }_{i}$ 是第 $i$ 个国家的确诊人数, Population ${ }_{i}$ 是第 $i$ 个国家的每 十万人口数, $P I_{i}$ 是第 $i$ 个国家每十万人的 COVID- 19 感染率; 公式 (6) (7) 是位序标准化的公式形式, 公式 (6) 中, $Y_{\mathrm{Score}_{i}}$ 是 $\mathrm{Score}_{i}$ 位序标准化化后的数据, $X_{\mathrm{Score}_{i}}$ 是 $\mathrm{Score}_{i}$ 升序的排序数, $N$ 是 $\mathrm{Score}_{i}$ 的总数或组数; 公式 (7) 中, $Y_{P I_{i}}$ 是 $P I_{i}$ 位序标准化化后的数据, $X_{P I_{i}}$ 是 $P I_{i}$ 升序的排序数, $N$ 是 $P I_{i}$ 的总数或组数。

\section{4 匹配性-恢复力分类体系}

本文利用匹配性指数和恢复力指数的数据分布特 征建立了匹配性-恢复力分类体系, 以 $\mathrm{SDR}=1 、 R=1$ 为 两条分界线,将 162 个国家分为 9 类 (图 1)。HH 型国 家加速行动供过于求的状态且恢复力较高, $\mathrm{MH}$ 型国家 加速行动供求相等且恢复力较高, LH 型国家加速行动 供小于求且恢复力较高, LM 型国家加速行动供小于求 且恢复力居中, MM 型国家加速行动供求相等且恢复力 居中, $\mathrm{HM}$ 国家加速行动供过于求且恢复力居中, $\mathrm{HL}$ 型 国家加速行动供过于求且恢复力较弱, ML 型国家加速 行动供求相等且恢复力较弱, LL 型国家加速行动供小 于求且恢复力较弱。

\section{5 相关性分析}

在获取各国可持续发展目标现状得分、各国可持续 发展目标加速行动数量的基础上,本文利用 SPSS 计算 可持续发展目标现状得分与可持续发展目标加速行动 数量的相关系数。加速行动数量与可持续发展目标得 分的相关系数大于 0 , 则正相关, 表示各国某项可持续 发展目标得分越高, 受到的可持续发展目标加速行动数 量越多; 反之, 则负相关, 表示各国某项可持续发展目标 得分越高, 受到的可持续发展目标加速行动数量越少。 鉴于预处理后的数据不呈现正态分布, 本文相关性分析

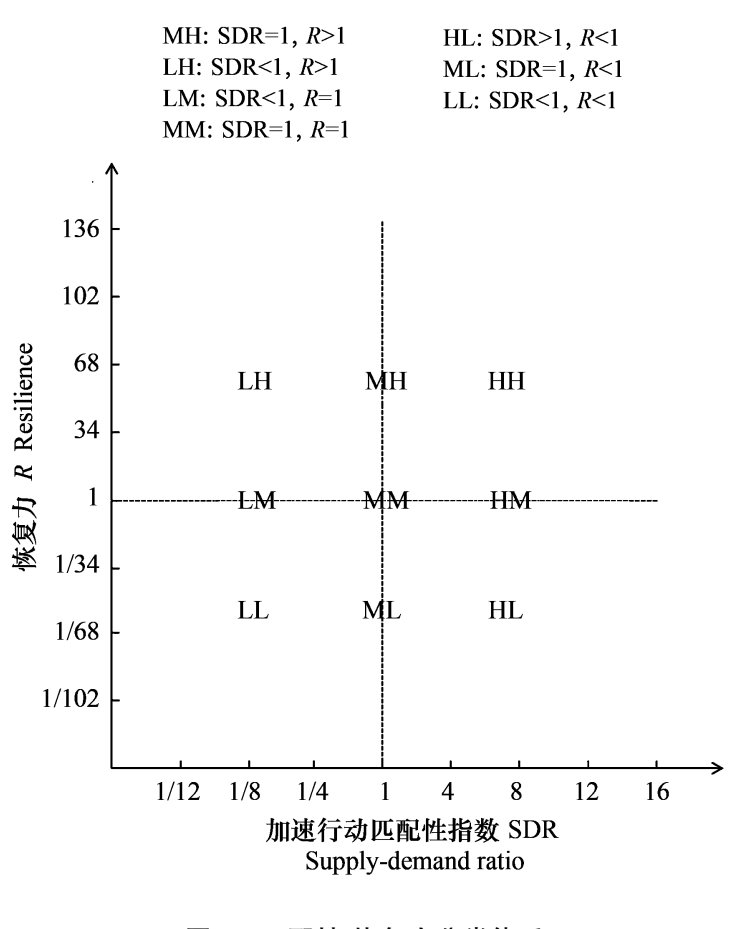

图 1 匹配性-恢复力分类体系

Fig.1 Matching-Resilience classification system

SDR: 加速行动匹配性指数 Supply-Demand Ratio; R: 恢复力 Resilience;HH: 高高 High-High; MH: 中高 Middle-High; LH: 低高 Low-High; LM : 低中 Low-Middle; MM : 中中 Middle-Middle; HM : 高 中 High-Middle; HL: 高低 High-Low; ML: 中低 Middle-Low ;LL: 低 低 Low-Low 
采取了 Spearman 相关分析方法,将 $P<0.05$ 认作为通过显著性检验。

\section{6 可持续发展目标关联网络分析}

由于一项可持续发展目标加速行动可以同时支持多个可持续发展目标,因此可以进一步基于可持续发展 目标加速行动政策文件探究各项可持续发展目标之间的关联关系。本文利用 Gephi 软件将各项可持续发展 目标之间的关系进行可视化,通过可持续发展目标加速行动政策文件中 17 项受益目标的两两共线频次, 来探 究政策文件中 17 项可持续发展目标之间的关联关系。Gephi 图中的点代表可持续发展目标 1-17,点的大 小,代表某可持续发展目标目标与其他可持续发展目标的关联性,点越大,代表该可持续发展目标与其他可持 续发展目标的联系越密切; 线代表两个可持续发展目标间的联系,线的粗细代表两个可持续发展目标的关联 度的大小, 线越粗联系越密切, 同时线颜色的深浅也代表着两个可持续发展目标的关联度的大小,颜色越深联 系越密切。

\section{2 结果分析}

2.1 加速行动匹配性指数 (SDR) 空间分布

现有可持续发展目标加速行动的实施与区域需求不匹配。如图 2, 可持续发展目标间的匹配性存在差异 性, 在 SDG3(良好健康与福祉, Good Health and Wellbeing) 中, 各国 SDR =1 的数量最多 (53 个), SDG8(体面 工作和经济增长, Decent Work and Economic Growth) 最少 (20个); 表明大部分国家在构建良好健康与福祉上 合理性较好,更多的人关注健康问题和国家之间人类福祉的公平性，但同时也可以看出国别之间经济增长的 不均衡性依然存在。在 SDG6 (清洁饮水和卫生设施, Clean Water and Sanitation) 中, 各国 SDR $<1$ 的情况最多 (74 个), 表明大部分国家对于解决清洁饮水和卫生设施问题的加速行动数量相对较少。在 SDG13 (采取紧 急行动应对气候变化及其影响, Climate Action) 中, 各国 SDR $>1$ 的情况最多 (73 个), 表明大部分国家对于解 决气候行动的加速行动数量过多, 导致过多加速行动实施的原因主要有两个:一是国际社会对气候变化后果 的认识逐渐加强, 二是实施的气候行动措施力度和效果没有达到预期要求, 导致大量相关加速行动的出现。

区域关注的可持续发展目标不同,并与其自然地理位置和社会发展水平有着密切的关系。亚洲国家大部 分处于上升发展期,基础建设、社会稳定、生产发展等方面可持续发展目标加速行动匹配性较好。欧洲国家生 产生活已经处于较高的水平, 更关注基础建设之外的目标, 如可持续发展, 气候行动,促进合作关系等方面的 可持续发展目标加速行动匹配性较好。而非洲国家则关注生产、防治荒漠化、制止和扭转土地退化现象方面。

加速行动的实施受限于可持续发展水平和国家经济基础,需要在发展中国家大力推动可持续发展加速行 动。在 17 项可持续发展目标中, 非洲、亚洲、大洋洲大部分国家 $\mathrm{SDR}<1$, 美洲、欧洲大部分国家 SDR $>1$ 。SDR 最小值主要出现在非洲地区 (50\%),SDR 最大值主要出现在美洲和欧洲地区 (36\%)。

2.2 恢复力指数 $(R)$ 空间分布

如图 3, $R>1$ 主要集中分布在欧洲国家, $R<1$ 主要集中分布在美洲国家。美洲 (78\%)、非洲 (74\%)、亚洲 (52\%) 区域大部分国家处于 $R<1$ 的状况, 说明美洲、非洲、亚洲区域从 COVID- 19 中恢复能力相对较低, 欧洲 (79\%)、大洋洲(100\%) 区域大部分国家处于 $R>1$ 状况, 说明欧洲、大洋洲区域从 COVID- 19 中恢复能力相对 较强。分析发现非洲国家平均可持续发展水平最低, 导致应对 COVID- 19 的恢复能力相对较低; 美洲平均可 持续发展水平较高, 但感染率高, 导致应对 COVID- 19 的恢复能力相对较低。欧洲各国平均可持续发展水平 最高, 导致应对 COVID- 19 的恢复能力相对较高; 大洋洲感染率最低, 导致应对 COVID- 19 的恢复能力相对较 高。中非共和国 (Central African Republic) 是恢复能力相对最低的国家, 原因在于它的可持续发展水平最低; 老挝 ( Lao PDR) 是恢复能力相对最高的国家, 原因在于它的感染率最低。虽然亚洲区域大部分国家 $(52 \%)$ 处 于 $R<1$ 的状况, 但在恢复能力前十的国家里, 亚洲国家占据 6 个, 原因在于它们的感染率较低。如恢复能力 排名第八的中国, 虽然初期疫情严重, 但是由于实施严格的防控措施, 在后期控制住了疫情, 同时可持续发展 水平较高, 导致对 COVID- 19 的恢复能力相对较高。美国的可持续发展水平尽管要高于中国,但感染率较高, 


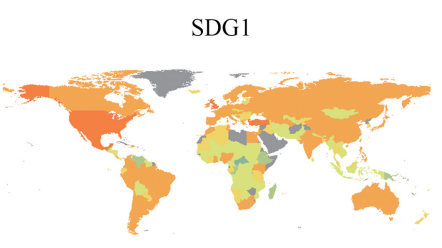

SDG4

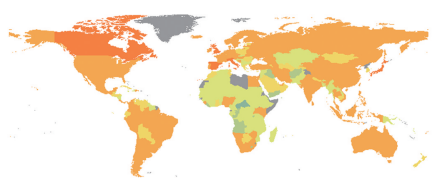

SDG7

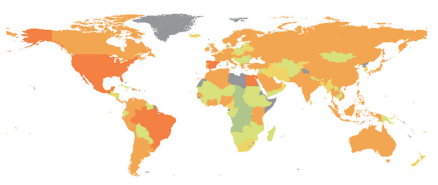

SDG10

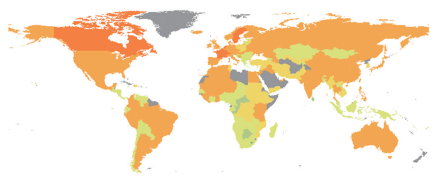

SDG13

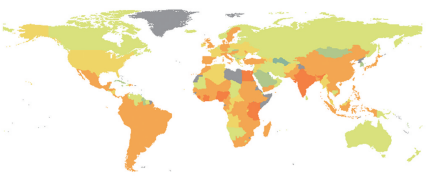

SDG16

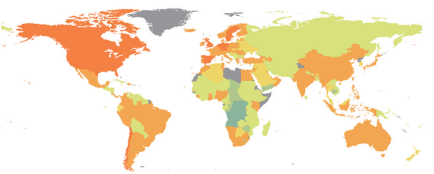

SDG2

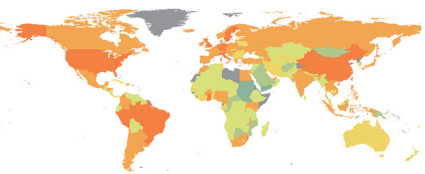

SDG5

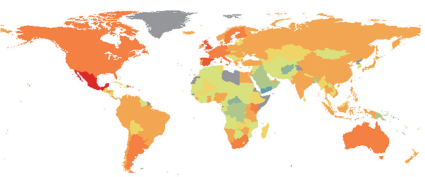

SDG8

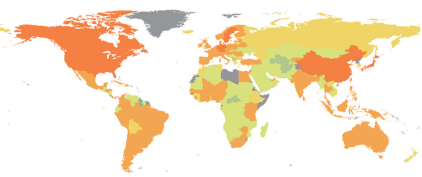

SDG11

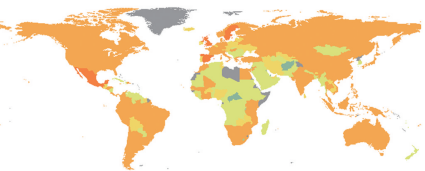

SDG14

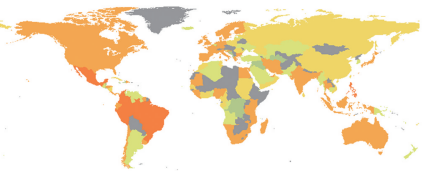

SDG17

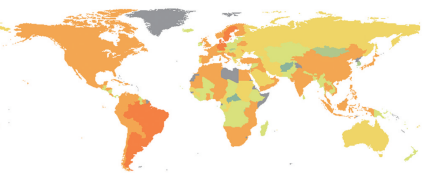

SDG3

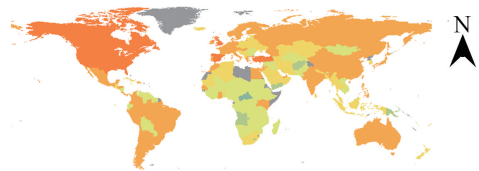

SDG6

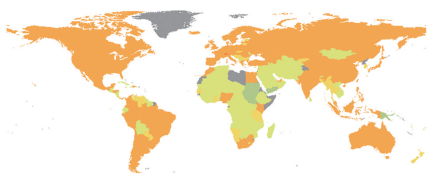

SDG 9

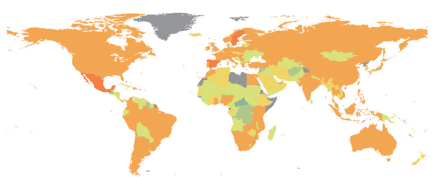

SDG12

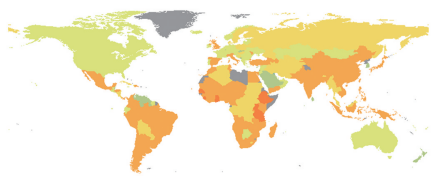

SDG15

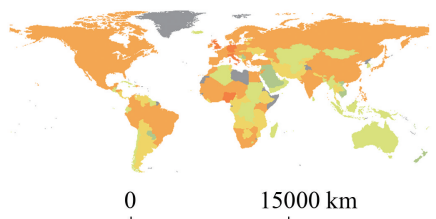

加速行动匹配性指数 SDR

\begin{tabular}{|l|l|}
\hline 无数据 & $\square$ \\
$0.01-0.08$ & \\
$0.01-00$ & $1.01-4.00$ \\
$0.09-0.13$ & $4.01-8.00$ \\
$0.14-0.25$ & $8.01-12.00$ \\
\hline $0.26-0.99$ & $12.01-16.00$ \\
\hline
\end{tabular}

图 2 各国 17 项可持续发展目标匹配性指数分布图

Fig.2 Distribution map of the matching index of SDG1-17 in various countries

SDG：可持续发展目标 Sustainable Development Goal; SDG1：无贫困 No poverty；SDG2：零饥饿 Zero Hunger; SDG3：良好健康与福祉 Good Health and Wellbeing; SDG4: 优质教育 Quality Education; SDG5: 性别平等 Gender Equality; SDG6: 清洁饮水和卫生设备 Clean Water and Sanitation; SDG7: 经济适用的清洁能源 Affordable and Clean Energy; SDG8: 体面工作和经济增长 Decent Work and Economic Growth; SDG9: 产业、创新和基础设施 Industry，Innovation and Infrastructure; SDG10: 减少不平等 Reduced Inequalities; SDG11：可持续城市和社区 Sustainable cities and communities; SDG12: 负责任消费和生产 Sustainable Consumption and Production; SDG13: 气候行动 Climate Action; SDG14: 水下生物 Life Under Water; SDG15: 陆地生物 Life on Land; SDG16: 和平、正义与强大机构 Institutions, good governance; SDG17: 促 进目标实现的伙伴关系 Partnerships for the goals

导致对 COVID-19 的恢复能力相对较低。

2.3 各国合理性-恢复力分类空间分布

大部分国家都集中在 HH、MH、LH、HL、ML、LL 六类里(图 4)。在大部分 SDG 目标 ( 76\%) 中, LL 型国家 占比最多, HH 型次之,表明大部分可持续发展目标的加速行动两极化现象严重。在 $82 \%$ 的可持续发展目标 
中,LL 型国家占比最多, 说明在这些可持续发展目标 中,大部分国家存在加速行动的合理性、应对 COVID-19 恢复能力差的问题。类型 HH、MH、LM 主要分布在欧 洲,类型 $\mathrm{LH}$ 主要分布在亚洲, 类型 MM、HM、ML、LL 主 要分布在非洲,类型 HL 主要分布在美洲。非洲各国主 要集中在类型 LL,处于恢复力低且合理性差的状态; 美 洲各国主要集中在类型 HL,处于恢复力低但 SDR 高的 状态;亚洲各国主要集中在类型 LH 和类型 LL, 属于 SDR 低的状态; 欧洲各国主要集中在类型 HH,处于恢 复力高且 SDR 高的状态, 大洋洲各国处于类型 LH, 处 于恢复力较高,但 SDR 较低的状态。

\section{4 可持续发展目标关联网络分析}

基于可持续发展目标加速行动政策文件对可持续

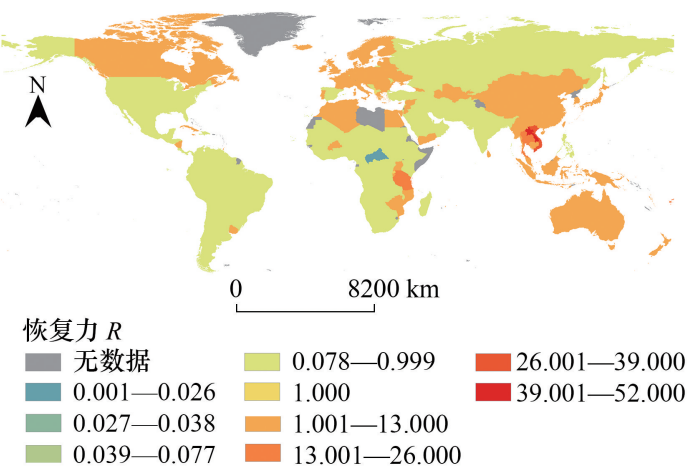

图 3 各国恢复力指数分布图

Fig.3 Distribution map of the resilience index of various countries 发展目标进行网络分析, 辨析可持续发展目标之间的关联关系。如图 5 所示, SDG13 与 SDG17, SDG5 与 SDG8,SDG12 与 SDG13 的关联度高, 表明一项加速行动的实施可以同时促进 SDG13 与 SDG17, SDG5 与 SDG8,SDG12 与 SDG13, 彼此之间的协同关系较强; SDG14 与各目标之间联系都不是很密切,下一步加速行动 需要单独加强 SDG14 的实施。

\section{3 讨论}

3.1 新冠疫情前各国加速行动数量与可持续发展水平的相关性

新冠疫情爆发前(数据搜集截至 2019.12.1) 实施的可持续发展目标加速行动数量与区域需求已存在不匹 配关系,并不能满足各国可持续发展目标的协同发展、整体实现。如表 1 所示, 17 项可持续发展目标中, SDG2、SDG5、SDG7、SDG8、SDG9 现状得分与加速行动数量呈显著正相关。说明在 2019 年联合国可持续发展 峰会后, 实施的加速行动大多作用于可持续发展目标现状较好的国家, 对于可持续发展目标现状较差的国家

表 1 现状得分-行动数量相关性系数表

Table 1 Correlation analysis between Goal Score and Action Number

\begin{tabular}{cc||cc}
\hline SDG & $\begin{array}{c}\text { 相关性系数 } \\
\text { Correlation coefficient }\end{array}$ & SDG & $\begin{array}{c}\text { 相关性系数 } \\
\text { Correlation coefficient }\end{array}$ \\
\hline SDG1 & -0.0501 & SDG10 & -0.0602 \\
SDG2 & $0.1791^{*}$ & SDG11 & 0.0303 \\
SDG3 & 0.1076 & SDG12 & -0.2255 \\
SDG4 & 0.1074 & SDG13 & 0.0927 \\
SDG5 & $0.2442^{* *}$ & SDG14 & 0.1048 \\
SDG6 & 0.0995 & SDG15 & 0.1435 \\
SDG7 & $0.2372^{* *}$ & SDG16 & 0.037 \\
SDG8 & $0.1822^{*}$ & SDG17 & -0.0745 \\
\hline
\end{tabular}

*在 0.05 水平 (双尾), 相关显著; **在 0.01 水平 (双尾), 相关显著; SDG : 可持续发展目标 Sustainable Development Goal; SDG1: 无贫困 No poverty; SDG2: 零饥饿 Zero Hunger; SDG3: 良好健康与福祉 Good Health and Wellbeing; SDG4: 优质教育 Quality Education; SDG5: 性别平等 Gender Equality; SDG6: 清洁饮水和卫生设备 Clean Water and Sanitation; SDG7: 经济适用的清洁能源 Affordable and Clean Energy; SDG8: 体面工 作和经济增长 Decent Work and Economic Growth; SDG9: 产业、创新和基础设施 Industry, Innovation and Infrastructure; SDG10: 减少不平等 Reduced Inequalities; SDG11: 可持续城市和社区 Sustainable cities and communities; SDG12: 负责任消费和生产 Sustainable Consumption and Production; SDG13: 气候行动 Climate Action; SDG14: 水下生物 Life Under Water; SDG15: 陆地生物 Life on Land; SDG16: 和平、正义与强大机 构 Institutions, good governance; SDG17: 促进目标实现的伙伴关系 Partnerships for the goals 
SDG1

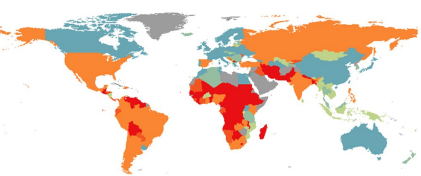

SDG4

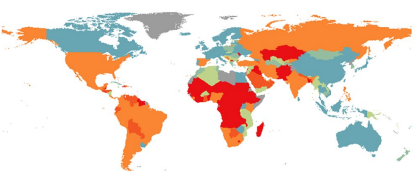

SDG7

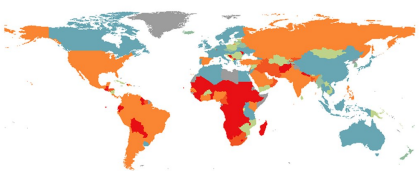

SDG10

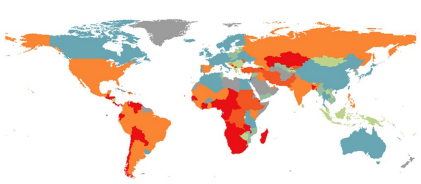

SDG13

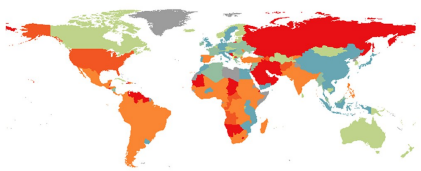

SDG16

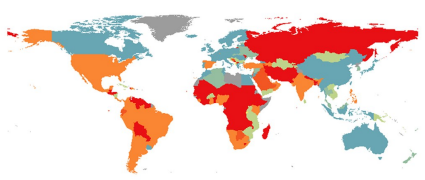

SDG2

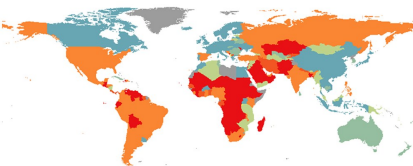

SDG5

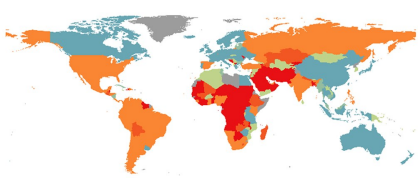

SDG8

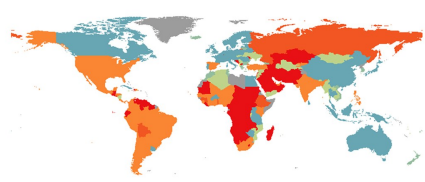

SDG11

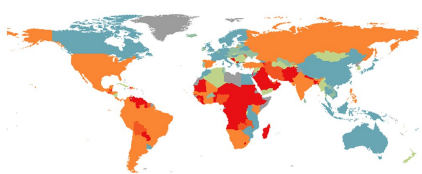

SDG14

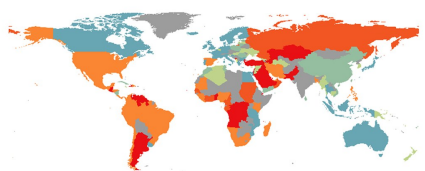

SDG17

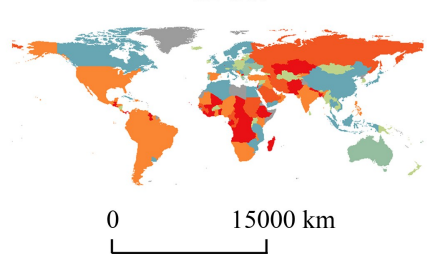

SDG3

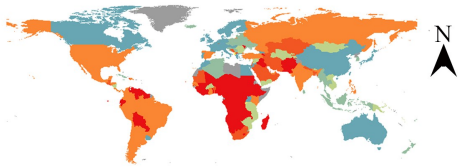

SDG6

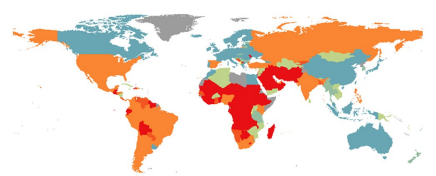

SDG9

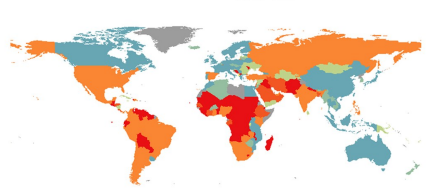

SDG12

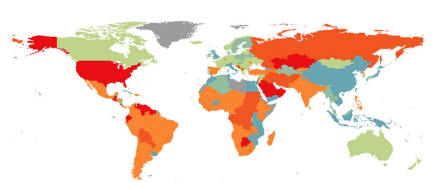

SDG15
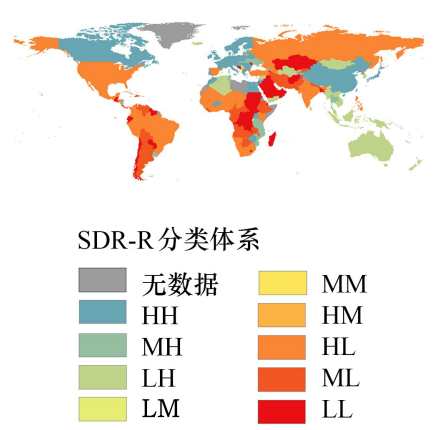

图 4 各国 17 项可持续发展目标类型分布图

Fig.4 Type distribution map of SDG1-17 in various countries

的实施力不足。加速行动主要来源于各国政府主动发起和多边组织机构、其他利益做关方自愿发起两大类。 $36 \%$ 的加速行动是各国政府颁布的政策,其余为其他组织机构和利益做关方。经济发展程度较好的国家政府 会颁布较多的加速行动, 经济发展程度较差的国家主要依赖于国际组织的帮助。各国可持续发展水平的异质 性导致可持续发展水平越高的国家往往拥有较多的加速行动数量,这部分不均衡是由国家自身的发展水平所 决定的。可持续发展目标现状较差的国家不能从多边组织机构和利益做关方发起的可持续发展目标加速行 动中获得足够的惠益, 国家间可持续发展水平差距与日俱增。下一步的加速行动应该更多考虑多边组织机构 和其他利益敒关方加速行动的合理性,加强建立国家之间的相互支持和协助机制 ${ }^{[31]}$, 形成多边合作, 实现互 惠共赢, 诸如一带一路、南南合作, 以加速行动供过于求的国家带动加速行动供小于求的国家, 实现全球加速 行动与区域需求匹配平衡,促进各国可持续发展目标的实现。

3.2 加速行动分类推动措施及研究不足

针对已经出现的不匹配现象,本文利用合理性-恢复力分类体系,结合可持续发展目标之间的关联关系， 对 9 类国家提出了下一步实施加速行动的主要方向。针对某项可持续发展目标,在分类体系中为 HH、HM 类 
的国家居于加速行动供过于求, 且恢复能力较强的状 态, 可以暂缓实施某项可持续发展目标加速行动的速 度,转而关注其他供小于求的可持续发展目标。MH、 $\mathrm{MM}$ 类国家居于加速行动供求相等, 且恢复能力较强的 状态, 不需要对相应的可持续发展目标过多关注,但是 也不需要暂缓对其实施的加速行动, 同时要关注其他供 小于求的可持续发展目标。LH、LM、LL 类国家居于加 速行动供不应求的位置, 要加强对相应可持续发展目标 实施加速行动的力度和数量, 同时注意提高 LL 类国家 的防疫水平和恢复能力, 可以在加速行动上面多实施有 关抗击疫情的措施。HL、ML 类国家均居于恢复能力较 差的位置, 要将加速行动实施的重点放在提高疫情恢复 力上,大力提高 SDG3 目标的实现。在具体的实施过程 中,要注意各国可持续发展目标之间的关联关系。 SDG8、SDG13、SDG17 与其他目标联系密切, 可以将这 三个目标作为枢纽, 深人探讨与其相连目标间的关系,

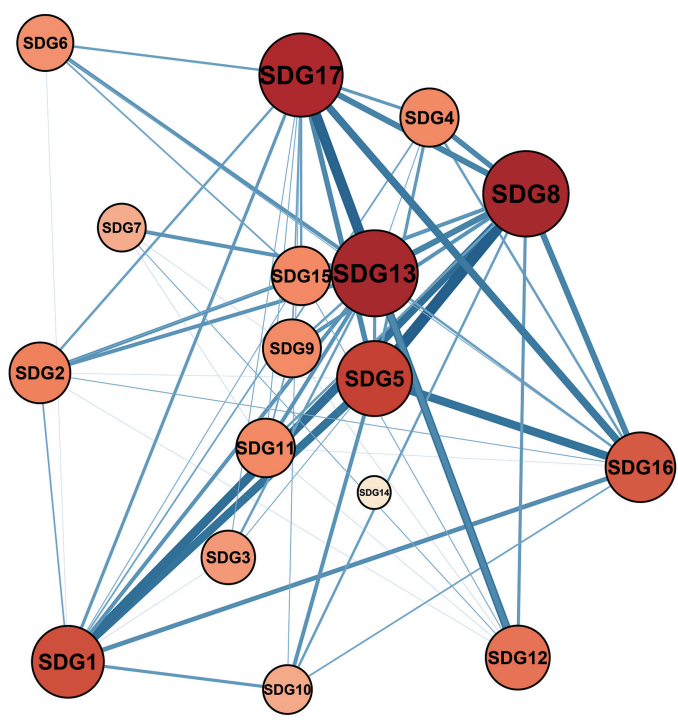

图 5 可持续发展目标 1-17 关联关系

Fig.5 Nexus relationship between SDG 1-17 发挥其纽带作用,协同促进各项可持续发展目标的实 现。此外,本文也有一定的局限性,全文是基于 “相对比较”的思想提出的研究框架,会随着其中某个国家的 改变而发生格局的改变,研究只能辨析出各国现有加速行动的相对匹配性和下一步加速行动的实施方向, 对 于动态化监测加速行动的实施起不到很好的效果。

下一步工作的重点将放在建立绝对评价的方法上,如结合可持续发展评估报告 (2020) 理清具体某项加 速行动的实施会带来多大幅度可持续发展得分的提升, 辨析不同加速行动的权重, 改进建立的匹配性指数模 型, 实现动态化监测。此外,在现有可持续发展目标评估的基础上, 以可持续发展目标得分距离最高得分的差 距作为生态系统服务的需求,生态系统的本底特征作为生态系统服务的供给, 探究生态系统服务与可持续发 展目标的关联关系, 以生态系统服务促进可持续发展目标的实现 ${ }^{[32-33]}$ 。

\section{4 结论}

本文对可持续发展评估报告和可持续发展目标加速行动等政策文件进行信息提取,建立了加速行动匹配 性指数模型和各国应对新冠疫情的恢复力指数模型,从政策文件分析的角度探究了截至 2020 年 8 月 28 日发 布的 214 项可持续发展目标加速行动数量与区域需求的匹配性和各国应对新冠疫情的恢复力,并根据匹配 性-恢复力分类体系将各国按照 17 项可持续发展目标分为 9 类,弥补了已有研究对于可持续发展目标的政策 分析呈现单目标分析且忽视了加速行动数量与区域需求匹配性的不足,为推动后疫情时代全球可持续发展目 标加速行动提供支撑。研究发现: (1) 现有可持续发展目标加速行动的实施与区域需求不匹配,这种不匹配 现象在 COVID- 19 爆发之前就已经出现; (2) 加速行动的实施受限于现有可持续发展水平和国家经济基础, 区 域关注的可持续发展目标与其自然地理位置和社会发展水平有着密切的关系,多边组织机构和其他利益敒关 方需要在发展中国家大力推动可持续发展加速行动; ( 3)下一步实施加速行动需要加强国际间的合作, 根据 分类框架和可持续发展目标的关联关系, 分重点推进加速行动的实施,完善可持续发展指标监测体系, 分类设 立后疫情时代不同时期的阶段目标, 分阶段循序渐进, 定期反馈追踪, 以在 2030 年促进 17 项可持续目标的 实现。

\section{参考文献 (References) :}

[ 1 ] Colglazier W. Sustainable development agenda: 2030. Science, 2015, 349(6252): 1048-1050. 
[ 2 ] Bain P G, Kroonenberg P M, Johansson L, Milfont T L, Crimston C R, Kurz T, Bushina E, Calligaro X, Demarque C, Guan Y J, Park J. Public views of the sustainable development goals across countries. Nature Sustainability, 2019, 2(9) : 819-825.

[ 3 ] Resasco D E, Wang B, Sabatini D. Distributed processes for biomass conversion could aid UN sustainable development goals. Nature Catalysis, 2018, 1(10): 731-735.

[ 4 ] Special Edition: Progress Towards the Sustainable Development Goals. (2019-05-08). https://unstats. un.org/sdgs/files/report/2019/secretarygeneral-sdg-report-2019--EN.pdf.

[ 5 ] 华廷, 赵文武. 加速推进可持续发展目标, 开启未来十年的行动和成就一一联合国可持续发展目标峰会介评. 生态学报, 2019, 39(20)： 7788-7791

[ 6 ] UN. SDGs Acceleration Actions. https://sustainabledevelopment.un.org/sdgactions.

[ 7 ] Thornton J. Covid-19 pandemic has derailed progress on sustainable development goals, says WHO. BMJ, 2020,369 : m1969.

[ 8 ] UN. Report of the Secretary-General, Economic and Social Council, UN. https://sustainabledevelopment.un.org/content/documents/26158Final_ SG_SDG_Progress_Report_14052020.pdf.(2021)

[ 9 ] Bill \& Melinda Gates. 2020 Goalkeepers Report: COVID- 19, A Global Perspective. [ 2020- 10-06 ]. https://www. gatesfoundation. org/ goalkeepers/downloads/2020-report/report_a4_en.pdf.(2021)

[10] Kumar P, Hama S, Omidvarborna H, Sharma A, Sahani J, Abhijith K V, Debele S E, Zavala-Reyes J C, Barwise Y, Tiwari A. Temporary reduction in fine particulate matter due to 'anthropogenic emissions switch-off' during COVID-19 lockdown in Indian cities. Sustainable Cities and Society, 2020, 62: 102382 .

[11] Ahmed M. Introduction to modern climate change. Andrew E. Dessler: Cambridge University Press, 2011 , 252 pp, ISBN- 10: 0521173159. Science of the Total Environment, 2020, 734: 139397.

[12] Naderipour A, Abdul-Malek Z, Ahmad N A, Kamyab H, Ashokkumar V, Ngamcharussrivichai C, Chelliapan S. Effect of COVID- 19 virus on reducing GHG emission and increasing energy generated by renewable energy sources: a brief study in Malaysian context. Environmental Technology \& Innovation, 2020, 20: 101151 .

[13] Le Quéré C, Jackson R B, Jones M W, Smith A J P, Abernethy S, Andrew R M, De-Gol A J, Willis D R, Shan Y L, Canadell J G, Friedlingstein P, Creutzig F, Peters G P. Temporary reduction in daily global $\mathrm{CO}_{2}$ emissions during the COVID- 19 forced confinement. Nature Climate Change, 2020, 10(7): 647-653.

[14] Wang Q Q, Lu M, Bai Z M, Wang K. Coronavirus pandemic reduced China's $\mathrm{CO}_{2}$ emissions in short-term, while stimulus packages may lead to emissions growth in medium-and long-term. Applied Energy, 2020, 278: 115735.

[15] Liu J G, Hull V, Godfray H C J, Tilman D, Gleick P, Hoff H, Pahl-Wostl C, Xu Z C, Chung M G, Sun J, Li S X. Nexus approaches to global sustainable development. Nature Sustainability, 2018, 1(9): 466-476.

[16] Pradhan P, Costa L, Rybski D, Lucht W, Kropp J P. A systematic study of sustainable development goal ( SDG) interactions. Earth's Future, 2017, 5(11): 1169-1179.

[17] Fu B J, Wang S, Zhang J Z, Hou ZQ, Li J H. Unravelling the complexity in achieving the 17 sustainable-development goals. National Science Review, 2019, 6(3): 386-388

[18] 张军泽, 王帅, 赵文武, 刘炎序, 傅伯杰. 可持续发展目标关系研究进展. 生态学报, 2019, 39(22): 8327-8337.

[19] Requejo-Castro D, Giné-Garriga R, Pérez-Foguet A. Data-driven Bayesian network modelling to explore the relationships between SDG 6 and the 2030 Agenda. Science of the Total Environment, 2020, 710: 136014.

[20] Zhu J, Cao Y, Zhai J Y, Zhao X, Zhao Y Q, Kang S H. Analysis on synergies and trade-offs in green building development: from the perspective of SDG 11. Chinese Journal of Population Resources and Environment, 2019, 17(4) : 341-351.

[21] Fader M, Cranmer C, Lawford R, Engel-Cox J. Toward an understanding of synergies and trade-offs between water, energy, and food SDG targets. Frontiers in Environmental Science, 2018, 6: 112.

[22] 朱婧, 孙新章, 何正. SDGs 框架下中国可持续发展评价指标研究. 中国人口 - 资源与环境, 2018,28(12): 9-18.

[23] 董仁才, 王韬, 张永霖, 张雪琦, 李欢欢. 我国城市可持续发展能力评估指标的元数据分析与管理. 生态学报, 2018 , 38 (11): 3775-3783.

[24] 陈军, 任惠茹, 耿雯, 彭舒, 叶芳宏. 基于地理信息的可持续发展目标(SDGs)量化评估. 地理信息世界, 2018, 25(1)：1-7.

[25] Bryan B A, Gao L, Ye Y Q, Sun X F, Connor J D, Crossman N D, Stafford-Smith M, Wu J G, He C Y, Yu D Y, Liu Z F, Li A, Huang Q X, Ren H, Deng X Z, Zheng H, Niu J M, Han G D, Hou X Y. China's response to a national land-system sustainability emergency. Nature, 2018, 559(7713): 193-204.

[26] Cohen M J. Let them eat promises: global policy incoherence, unmet pledges, and misplaced priorities undercut progress on SDG 2. Food Ethics, $2019,4(2): 175-187$

[27] Rozhenkova V, Allmang S, Ly S, Franken D, Heymann J. The role of comparative city policy data in assessing progress toward the urban SDG targets. Cities, 2019, 95: 102357.

[28] Fu B J, Zhang J Z, Wang S, Zhao W W. Classification-coordination-collaboration: a systems approach for advancing Sustainable Development Goals. National Science Review, 2020, 7( 5): 838-840.

[29] 刘建国, Hull V, Batistella M, DeFries R, Dietz T, 付峰, Hertel T W, Izaurralde R C, Lambin E F. 远程耦合世界的可持续性框架. 生态学 报, 2016, 36(23): 7870-7885.

[30] UN. Sustainable Development Report 2019. https://www.sdgindex.org/.

[31] Sachs J D, Schmidt-Traub G, Mazzucato M, Messner D, Nakicenovic N, Rockström J. Six transformations to achieve the sustainable development goals. Nature Sustainability, 2019, 2(9): 805-814.

[32] Caichun Yin, Wenwu Zhao, Francesco Cherubini, Paulo Pereira. Integrate ecosystem services into socio-economic development to enhance achievement of sustainable development goals in the post-pandemic era. Geography and Sustainability, 2021, 2(1):68-73.

[33] Siqi Yang, WenwuZhao, YanxuLiu, Francesco Cherubini, Bojie Fu, Paulo Pereira. Prioritizing sustainable development goals and linking them to ecosystem services: An expert's knowledge evaluation. Geography and Sustainablity, 2020, 4(1) 321-330 